\title{
Fixação iriana de lentes de câmara posterior para o tratamento da afacia
}

\author{
Iris fixation of posterior chamber intraocular lenses \\ for the treatment of aphakia
}

Ana Luiza Lise Ferreira ${ }^{1}$, Leonardo Verri Paulino ${ }^{2}$, Jose Ricardo Carvalho Lima Rehder ${ }^{3}$

\section{Resumo}

Objetivo: avaliar os resultados de uma série de casos de fixação iriana de lentes intraoculares de câmara posterior para correção de afacia, realizados no setor de catarata da disciplina de Oftalmologia da Faculdade de Medicina do ABC e compará-los a literatura existente. Métodos: estudo prospectivo de oito pacientes afácicos, que apresentavam ausência de suporte capsular adequado e foram submetidos à cirurgia para implante secundário de LIO de câmara posterior com fixação iriana seguindo a técnica modificada de McCannel. Resultados: sete dos oito pacientes (87,5\%) que não tiveram complicações cirúrgicas obtiveram melhora na acuidade visual corrigida. Um paciente $(12,5 \%)$ apresentou complicação intra-operatória, evoluindo com ceratopatia bolhosa do pseudofácico. Um paciente (12,5\%) apresentou glaucoma secundário à dispersão pigmentar. Conclusão: em nosso estudo a técnica de fixação iriana de câmara posterior se mostrou eficaz e segura para o tratamento da afacia, em concordância com os dados da literatura.

Descritores: Afacia; Implante de lente intra-ocular/métodos;Técnicas de sutura; Cápsula do cristalino/patologia

\footnotetext{
'Estagiário da Disciplina de Oftalmologia da Faculdade de Medicina do ABC - FMABC - Santo André (SP), Brasil. ${ }^{2}$ Médico colaborador da Disciplina de Oftalmologia da Faculdade de Medicina do ABC - FMABC - Santo André (SP), Brasil. ${ }^{3}$ Professor Titular da Disciplina de Oftalmologia da Faculdade de Medicina do ABC - FMABC - Santo André (SP), Brasil. 


\section{INTRODUÇÃO}

A s lentes intra-oculares (LIOs) historicamente têm sido utilizadas para substituir o cristalino após a cirurgia de catarata ${ }^{1}$.

$\mathrm{Na}$ ausência de suporte capsular adequado que impeça o implante da LIO no saco ou sulco capsular, a mesma pode ser implantada na câmara anterior (CA) ou posterior $(\mathrm{CP})$ através de diferentes técnicas. Na câmara anterior existe a opção de implante de LIO com hastes flexíveis abertas apoiadas no ângulo, LIO de captura iriana (íris claw) e LIO de fixação retro-pupilar. $\mathrm{Na}$ câmara posterior as opções consistem em fixar a LIO à esclera e à íris ${ }^{2}$.

Por um longo período de tempo as LIOs de câmara anterior (CA) foram o tipo predominantemente utilizado para o tratamento da afacia na ausência de suporte capsular. Nos anos 80, tornou-se evidente que as LIOS de CA estavam associadas com várias complicações como perda irreversível de células endoteliais levando à ceratopatia bolhosa, seqüelas inflamatórias intratáveis, edema macular cistóide, dano das estruturas angulares, sinéquias anteriores, bloqueio pupilar, atrofia de íris e hifema ${ }^{3}$. Atualmente com novo design, as LIOs de CA têm ressurgido como opção terapêutica.

Técnicas utilizando sutura para fixar lentes de câmara posterior foram então desenvolvidas. Em 1976, McCannel descreveu o uso de suturas de fixação uveal para estabilizar as LIOs de câmara posterior ${ }^{4}$. Em 1994 Siepers a aprimorou, possibilitando a confecção do nó sem a necessidade da abertura da câmara anterior e exposição iriana ${ }^{5}$. Tal técnica, associada a um método de fixação iriana de LIO dobrável através de uma pequena incisão corneana descrita por Condon em 2003, eliminou a necessidade de incisões maiores que as necessárias nas cirurgias atuais de catarata, minimizando o trauma cirúrgico ${ }^{6}$.

As fixações esclerais foram desenvolvidas mais recentemente em relação às fixações irianas. Malbran et al. foi o primeiro a descrever a fixação escleral transsulco em afácicos ${ }^{7}$.

Apesar da fixação escleral ser tecnicamente menos exigente em relação à fixação iriana, esta associada a um risco maior de formação de sinéquia anterior periférica, glaucoma e hemorragia intra-ocular pela passagem da agulha e sutura através do corpo ciliar. Complicações tardias estão relacionadas à erosão da sutura através da esclera e conjuntiva que podem secundariamente levar a endoftalmite, por promover uma rota de entrada dos microorganismos da superfície ocular ${ }^{8}$.
A vantagem da técnica de fixação iriana inclui uma correção óptica perto do ponto nodal, menor risco de sinéquia periférica anterior, um menor risco de lesão endotelial (quando comparada com as LIOs-CA) e a possibilidade de estabilização da lente pelo suco ciliar. Como não se move muito durante a flutuação do tamanho da pupila, a íris periférica consiste em uma plataforma segura para a fixação da sutura ${ }^{8}$.

A seleção da LIO e a técnica empregada para a correção de afacia em pacientes com ausência de apoio capsular adequado continuam a ser uma controversa. As estruturas angulares, a anatomia da íris, a presença de antecedentes de glaucoma e a idade, são considerações importantes que devem ser feitas na seleção apropriada do método e da técnica de fixação. ${ }^{(9)}$

O objetivo deste trabalho é avaliar os resultados de uma série de casos de fixação iriana de câmara posterior em pacientes afácicos e compará-los a literatura existente.

\section{Métodos}

Em um estudo prospectivo, aprovado pelo comitê de ética e pesquisa da Faculdade de Medicina do ABC, foram selecionados oito pacientes do Ambulatório de Catarata do Instituto de Olhos da Faculdade de Medicina do $\mathrm{ABC}$, que foram submetidos à cirurgia de catarata há pelo menos três meses e não tiveram a lente intraocular implantada no ato cirúrgico devido a complicações intra-operatórias com ausência de suporte capsular adequado.

Estes pacientes, após o consentimento informado, foram submetidos à avaliação clínica pré-operatória, e avaliação oftalmológica completa incluindo potencial de acuidade visual. Foram estabelecidas como critério de exclusão outras morbidades oculares associadas (glaucoma, doença retiniana ou corneana), íris incompatível com o procedimento (discoria, desinserção ou atrofias setoriais importantes) e ausência de prognóstico de melhora da acuidade visual.

O poder da lente intra-ocular (LIO) foi calculado para a colocação no saco capsular sem nenhum ajuste no poder, utilizando-se biometro (Ocuscan $\mathrm{RXP}^{\circledR}$ Alcon) pela técnica de contato e fórmula SRK-T.

Após a anestesia peribulbar, é confeccionada a incisão principal tipo clear córnea de 3,5 mm e infundido cloreto de carbacol $0,1 \mathrm{mg} / \mathrm{ml}$ na CA para produzir miose.

Quando necessário,é realizado vitrectomia anterior/posterior com o objetivo de propiciar um espaço 


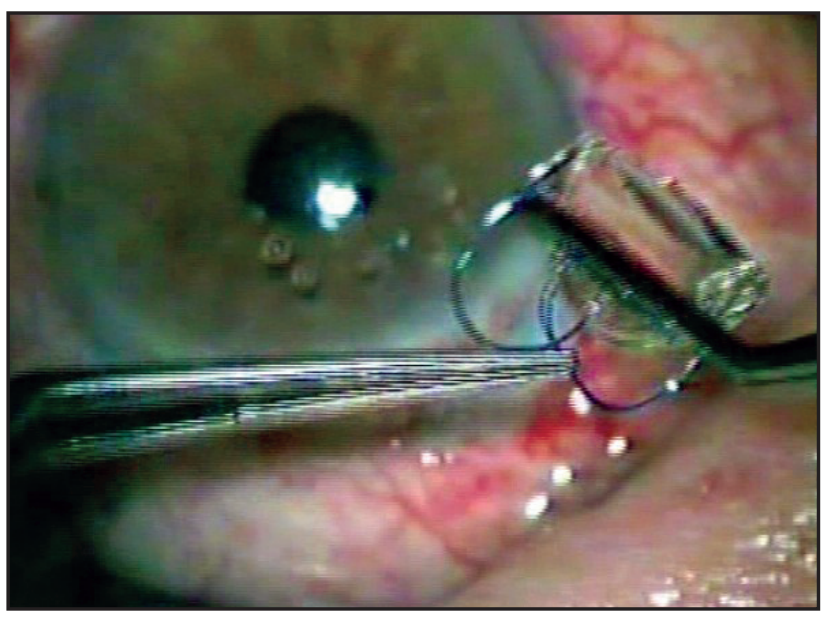

Figura 1. LIO com a parte óptica dobrada em seu meridiano horizontal.

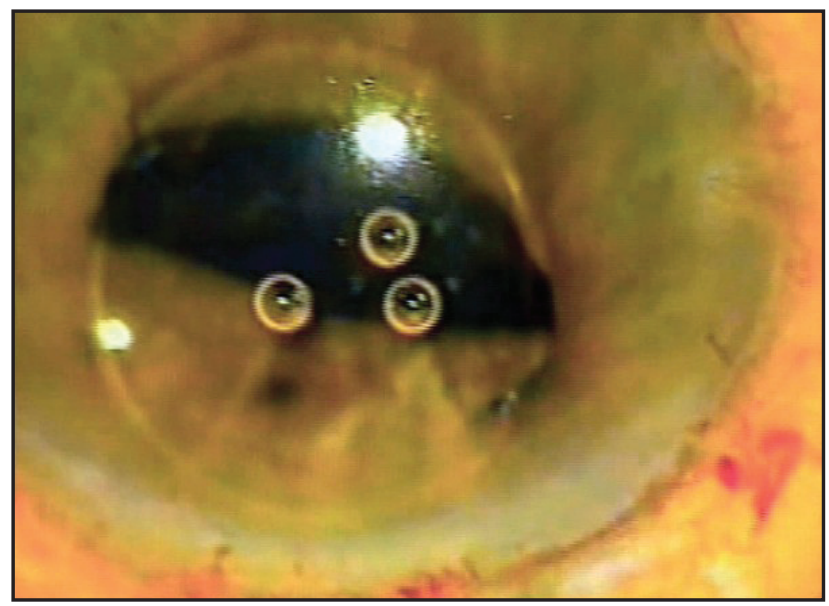

Figura 3. Captura pupilar estabilizando a óptica.

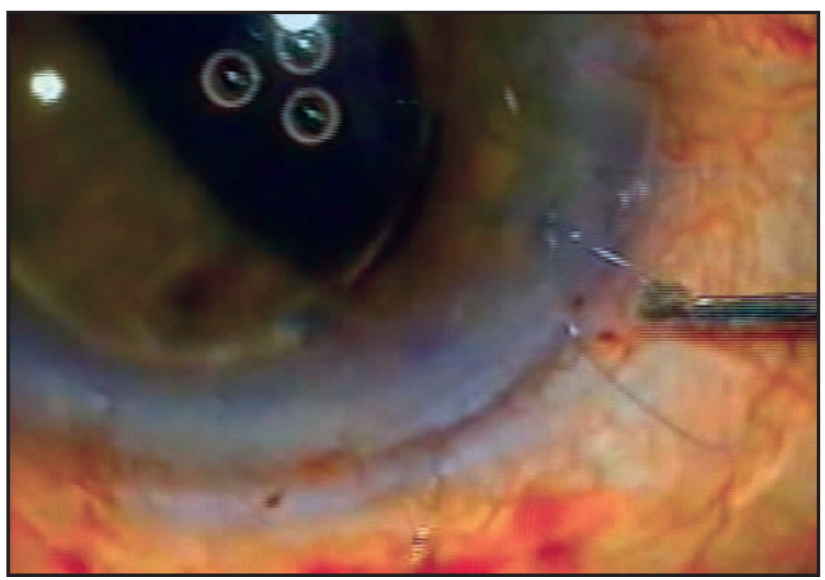

Figura 5. Fios recuperados pela paracentese com o auxílio de ganchos de Kuglen, para a confecção do nó

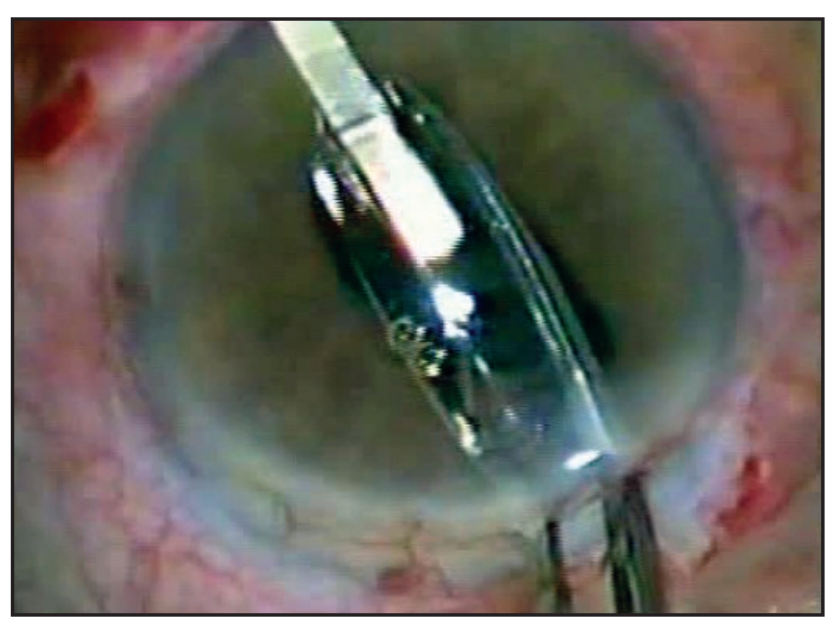

Figura 2. Alças orientadas verticalmente e projetadas atrás da íris. A espátula é introduzida dentro da zona óptica, acima da pupila.

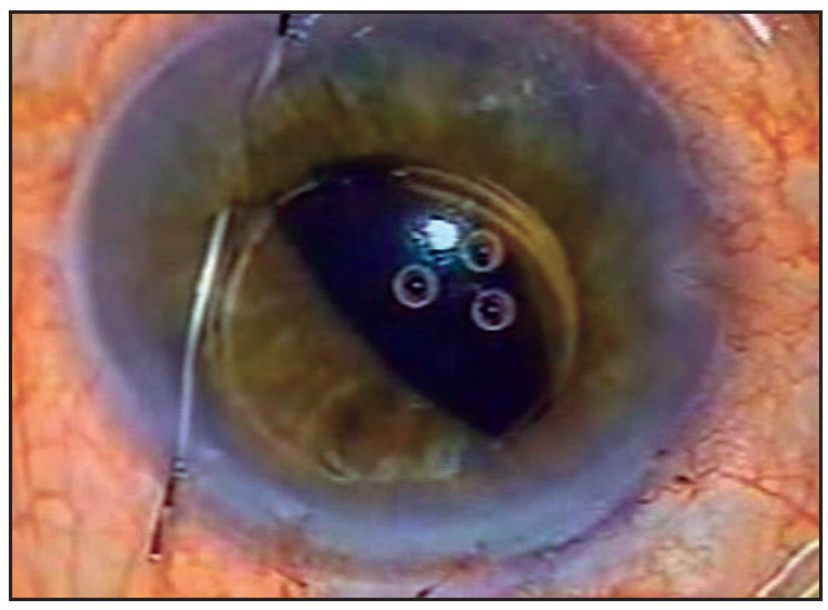

Figura 4. Sutura com fio de polipropileno $10-0$ passado via paracentese pela meia periferia iriana, atrás da alça, retornando através da íris e saindo pela córnea distal

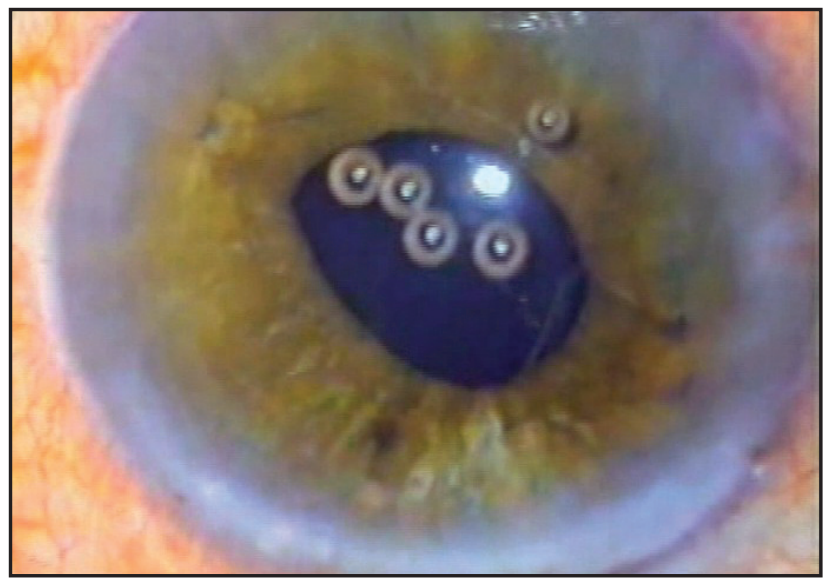

Figura 6. Parte óptica inserida na câmara posterior após as suturas 
retropupilar antes do implante da LIO.

Uma paracentese acessória é criada a $180^{\circ}$ da incisão principal.

Uma LIO 3-peças acrílica dobrável (Sensar AR40 - AMO, TYPE7B -Alcon, MA60AC - Alcon), é dobrada em seu meridiano horizontal ( $3 \mathrm{hs}$ - 9hs), com o cuidado de manter as alças englobadas pelas partes ópticas (Figura 1).

Com o auxílio de pinça de Buratto é realizado o implante da lente mantendo as duas alças projetadas atrás da íris e a parte óptica no plano logo acima, uma espátula de íris é introduzida pela paracentese acessória e locada posteriormente à parte óptica da LIO entre os pólos das alças (Figura 2).

A LIO é lentamente desenrolada com as alças se extendendo por trás da íris, enquanto a óptica, apoiada sobre a espátula, permanece temporariamente capturada e estabilizada pela pupila (Figura 3 ).
Inicialmente, a sutura modificada de McCannel é utilizada para fixar cada alça da LIO à meia periferia da íris, com fio polipropileno 10-0 com agulha longa e curva do tipo PC-7 (Alcon). Com auxilio do gancho de Kuglen, os fios são levados às incisões acessórias previamente confeccionadas perpendicularmente à passagem da agulha e, através da técnica de Siepser, confeccionado o nó para fixar as alças (Figuras 4 e 5).

Para completar a inserção, a zona óptica é inserida na câmara posterior (Figura 6).

No pós-operatório os pacientes são medicados com Acetato de Prednisona 0,5mg/kg/dia VO, Acetato de Prednisolona ${ }^{\circledR} 1 \%$ colírio de $3 / 3$ hs até retirada seguindo critério médico, Gatifloxacina ${ }^{\circledR}$ de $6 / 6$ hs por 7 dias e Cetorolac de Trometamina $0,5 \%{ }^{\circledR}$ de $8 / 8$ hs por pelo menos 7 semanas.

A retirada de pontos e refração é preconizada após sete semanas de cirurgia.

Tabela 1

Sexo, idade, olho operado e período de acompanhamento

\begin{tabular}{lcccc}
\hline Pacientes & Sexo & Idade(anos) & Olho operado & $\begin{array}{c}\text { Acompanhamento } \\
\text { (meses) }\end{array}$ \\
\hline Paciente 1 & Feminino & 67 & Direito & 18 \\
Paciente 2 & Masculino & 87 & Esquerdo & 18 \\
Paciente 3 & Masculino & 56 & Direito & 16 \\
Paciente 4 & Masculino & 99 & Direito & 10 \\
Paciente 5 & Masculino & 62 & Direito & 10 \\
Paciente 6 & Feminino & 88 & Esquerdo & 10 \\
Paciente 7 & Feminino & 79 & Esquerdo & 10 \\
Paciente 8 & Feminino & 55 & Direito & 8 \\
\hline
\end{tabular}

Tabela 2

Acuidade visual com (C/C) e sem correção (S/C), no pré-operatório e pós-operatório final, modelo de LIO e complicações intra-operatórias

\begin{tabular}{|c|c|c|c|c|c|c|}
\hline \multirow[t]{2}{*}{ Pacientes } & \multirow{2}{*}{$\begin{array}{c}\text { Pré- operatório } \\
\text { S/C }\end{array}$} & \multirow[b]{2}{*}{$\mathrm{C} / \mathrm{C}$} & \multirow[t]{2}{*}{ Modelo LIO } & \multirow[t]{2}{*}{ Complicações } & \multicolumn{2}{|c|}{ Pós-operatório } \\
\hline & & & & & $\mathrm{S} / \mathrm{C}$ & $\mathrm{C} / \mathrm{C}$ \\
\hline 1 & CD $30 \mathrm{~cm}$ & $20 / 40$ & AR40 & s/ complicações & $20 / 80$ & $20 / 40$ \\
\hline 2 & $\mathrm{CD} 10 \mathrm{~cm}$ & $20 / 80 p$ & AR40 & s/ complicações & $20 / 100$ & $20 / 50$ \\
\hline 3 & CD 2 metro & $20 / 40$ & AR40 & $\begin{array}{c}\text { Desincerção } \\
\text { alça-ótica }\end{array}$ & $\mathrm{CD} 1 \mathrm{~m}$ & $\mathrm{~S} / \mathrm{M}$ \\
\hline 4 & $\mathrm{CD} 30 \mathrm{~cm}$ & $20 / 100$ & TYPE7B & $\begin{array}{c}\text { Rachadura } \\
\text { na ótica } 2 \text { LIOs }\end{array}$ & $20 / 63$ & $20 / 40$ \\
\hline 5 & CD 2 metros & $20 / 50$ & MA60AC & s/ complicações & $20 / 25 p$ & $20 / 20$ \\
\hline 6 & CD 2 metros & $20 / 30$ & MA60AC & s/ complicações & $20 / 50$ & $20 / 20$ \\
\hline 7 & CD $30 \mathrm{~cm}$ & $20 / 50$ & MA60AC & s/ complicações & $20 / 80$ & $20 / 30$ \\
\hline 8 & CD 2 metros & $20 / 30$ & MA60AC & s/complicações & $20 / 40$ & $20 / 25$ \\
\hline
\end{tabular}


Os dados analisados ao fim do acompanhamento foram: acuidade visual pré-operatória e pós-operatória final, com e sem correção e complicações intra e pósoperatórias.

\section{Resultados}

Oito olhos de 8 pacientes afácicos que preencheram os critérios de inclusão e exclusão, foram submetidos à fixação iriana de LIO de câmara posterior com sucesso, cinco tiveram o procedimento realizado em olho direito $(62,5 \%)$ e três em olho esquerdo $(37,5 \%)$, com acompanhamento de 12,1 \pm 5 meses. Média de idade de $73 \pm 18$ anos. Quatro pacientes eram homens (50\%) e quatro mulheres (50\%) (Tabela 1$)$.

Quatro pacientes tiveram implantadas LIOs MA60AC (Alcon), três Sensar AR40 (AMO) e um Type 7B (Alcon) (Tabela 2).

Duas $(25 \%)$ das 8 cirurgias apresentaram complicações intra-operatórias, ambas relacionadas com a LIO. O paciente 3, apresentou desinserção alça-ótica no momento do implante da lente (Sensar AR40 - AMO) sendo necessário ampliação da incisão e substituição da mesma, evoluindo no pós-operatório com ceratopatia bolhosa do pseudo-fácico. O paciente 4 apresentou como intercorrência rachadura na região central da ótica das duas LIOs iniciais (Type7B - Alcon), sendo necessário o implante de uma terceira lente, do mesmo modelo, dobrando-a o mínimo possível. Evoluiu no pós-operatório com aumento da pressão intra-ocular controlada com medicamentos tópicos antiglaucomatosos (Tabela 2).

Nenhum paciente apresentou descentralização da LIO, ruptura, desinserção da sutura iriana, formação de sinéquia anterior periférica sobrejacente à região da sutura, hemorragia vítrea, edema macular cistóide, ou demais alterações retinianas.

Dos oito pacientes, apenas um (12,5\%) (paciente 3) não apresentou melhora na acuidade visual após a cirurgia devido à ceratopatia bolhosa. Dos 7 (87,5\%) restantes, todos apresentaram melhor acuidade visual corrigida igual ou superior à 20/50, destes. quatro (50\%) com visão melhor ou igual à 20/30 (Tabela 2).

\section{Discussão}

Existem poucos grandes estudos que abordam a fixação iriana de câmara posterior. Muitos destes incluem técnicas antigas e mais complexas.

Em 1994, utilizando uma grande incisão limbar $(7 \mathrm{~mm})$, LIO rígida de câmara posterior com 4 fenestrações na ótica e sutura na meia periferia iriana, Navia-Aray reportou excelentes resultados visuais nos quais $93 \%$ dos pacientes apresentaram acuidade visual igual ou superior à pré-operatória, sendo 63\% 20/40 ou melhor, sem a ocorrência de complicações significativas no segmento anterior em 30 olhos. ${ }^{(10)}$ Quatro pacientes apresentaram discreta dispersão de pigmento e um evoluiu com edema macular cistóide persistente. Nenhum paciente desenvolveu edema corneano, tilt ou subluxação da LIO, irite, endoftalmite ou descolamento de retina em um acompanhamento de 40 meses.

Hoh et al reportaram uma melhora na acuidade visual após técnica de fixação iriana com LIO de $7 \mathrm{~mm}$ e 2 fenestrações na ótica em 27 dos 30 olhos, sem maiores complicações ${ }^{11}$.

Zeh et Price utilizando a mesma lente e uma técnica de fixação através de incisão limbar conseguiram $82,1 \%$ de acuidade visual igual ou superior a 20/80 e $57,1 \%$ 20/40, ou melhor ${ }^{12}$.

Destes estudos, complicações relacionadas à proximidade da LIO a íris posterior incluindo dispersão de pigmento, evolução de glaucoma, irites crônica e edema macular cistóide parecem ser mínimas e não superiores a outras técnicas de fixação ${ }^{13}$.

Em 2003 associando uma pequena incisão corneana à técnica de McCannel modificada por Siepers, que possibilita a confecção do nó sem a abertura da câmara anterior ${ }^{5}$, Condon tornou mais simples a fixação iriana de LIOs de câmara posterior por permitir a realização da técnica cirúrgica em um sistema fechado, com menor flutuação da pressão intra-ocular sem a necessidade de grandes incisões corneanas ou limbares ${ }^{6}$.

Em nosso estudo optamos por esta técnica pelas seguintes vantagens: posicionamento seguro da LIO durante a passagem da sutura devido à captura óptica, ausência de contato entre a sutura e a ótica, cuja fricção com o polimetilmetacrilato (PMMA) poderia resultar em uma quebra tardia da sutura, passagem da agulha pela periferia iriana cujos vasos radiais encapsulados e de menor calibre diminuem o risco de sangramento.

Uma das preocupações com esta técnica consiste no risco de uma captura óptica inadequada pela íris levando a perda da LIO posteriormente durante a confecção da sutura. Uma estratégia para diminuir o risco foi a utilização de espátula de íris sob a LIO durante sua abertura até a estabilização da ótica sobre a iris. Também foi utilizado cloreto de carbacol $0,1 \mathrm{mg} / \mathrm{ml}$ no intra-operatório para minimizar o efeito midriático causado pela anestesia, garantindo a miose necessária para uma captura óptica eficaz.

Das 8 cirurgias, nenhuma apresentou captura 
óptica inadequada. Em 3 cirurgias (37,5\%) foi necessário repassar um dos fios, uma delas devido à pega ineficaz da alça durante a primeira tentativa, outra pelo não deslizamento do nó através da paracentese acessória, e a terceira pela perda da pega de uma das alças, secundária a uma discreta mobilização da LIO ao passar o fio na alça contra lateral.

Todas as passagens da agulha pela íris ocorreram sem intercorrência, não apresentando sangramento durante todo o processo de sutura.

Vitrectomia anterior e retropupilar podem ser necessárias quando há presença de vítreo que possa dificultar o implante da LIO. Neste estudo, em 5 dos 8 pacientes $(62,5 \%)$ foi realizado vitrectomia anterior com tesoura de Vannas para liberação de pequenas traves vítreas.

A descentralização da LIO é uma complicação presente em todas as técnicas citadas. A causa da descentralização na técnica modificada de McCannel de fixação iriana com a presença de sutura intacta ainda não é clara. Levantam-se as seguintes possibilidades: sutura ineficientemente bem travada, captura excessiva de íris durante a passagem da agulha, formando excesso de tecido que impediria uma tensão adequada sobre a alça e uma desigualdade dos vetores de força entre as duas fixações. No entanto, suturas demasiadamente fortes podem resultar em uma proximidade muito grande entre a óptica da lente e a íris resultando em um aumento da dispersão de pigmentos irianos ${ }^{14,15}$.

Em um estudo que relata 4 casos de complicações relacionadas a esta técnica, 3 deles se devem à descentralização da LIO com sutura intacta ${ }^{(14)}$. Em outro trabalho retrospectivo em que são analisados a aplicação desta técnica em 46 pacientes, apenas 2 apresentaram tal complicação, um por afrouxamento da sutura e o outro por desinserção alça-ótica ${ }^{10}$.

Neste estudo, nenhum dos 8 pacientes apresentaram descentralização da LIO, porém em 1 (12,5\%) ocorreu desinserção alça-ótica no momento do implante da lente.

Na literatura pesquisada não há citação de complicações intra-operatórias envolvendo quebra ou rachadura da LIO. No entanto neste trabalho, duas lentes apresentaram rachadura da ótica, secundária ao dobramento com pinça, sendo ambas Type7B - Alcon. Nenhum dos estudos relata experiência com este tipo de LIO. Nas fixações irianas que empregam a mesma técnica que utilizamos, há referencias de LIOs MA60AC - Alcon 6,10,14 e AR40 - AMO ${ }^{16}$ que em nossas cirurgias também não apresentaram tal complicação.

Embora glaucoma não seja uma complicação fre- qüente, ela pode acontecer principalmente secundária a dispersão pigmentar ${ }^{(6)}$. Em nosso estudo, 1 paciente $(12,5 \%)$ necessitou de medicação tópica antiglaucomatosa para controle da pressão intra-ocular (PIO). No estudo citado anteriormente, dos 46 pacientes submetidos à fixação iriana, três apresentaram dispersão pigmentar, no qual 1 necessitou de medicação para controle da (PIO) ${ }^{6}$.

Em relação à acuidade visual, os 7 pacientes (87,5\% ) que não apresentaram complicações intra-operatórias, obtiveram melhora devido ao tratamento da afacia.

Em nosso estudo a técnica de fixação iriana de câmara posterior se mostrou eficaz e segura para o tratamento da afacia, em concordância com os dados da literatura. No entanto, a lente intra-ocular Type7B-Alcon, não se mostrou apropriada para o uso nesta técnica.

\section{Abstract}

Purpose: evaluate the results of a case series of iris-sutured posterior chamber IOLs for the correction of aphakia in the cataract sector of the department of Ophthalmology of the ABC School of Medicine and compare them with the existing data. Methods: prospective study of eight patients who underwent foldable IOL implantation using modified McCannel iris suture fixation for aphakia in the absence of capsule support. Results: seven of eight patients (87,5\%) without surgical complications improved their best corrected visual acuity. One patient (12,5\%) with surgical complication developed pseudophakic bullous keratopathy. One patient (12,5\%) developed secondary glaucoma caused by dispersion syndrome. Conclusion: in our study the iris-sutured posterior chamber IOLs prove to be safe and effective in the treatment of aphakia, in accordance with existing data.

Keywords: Aphakia; Lens implantation, intraocular/methods; Suture techniques; Lens capsule, crystalline/pathology

\section{REFERÊNCIAS}

1. RidleyH. Late surgical results of use of the intraocular acrylic lens. J Int Coll Surg. Sep 1956; 26(3):335-41

2. Dick HB, Augostin AJ. Lens implant selection with abscense of capsular support. Curr Opin Ophthalmol. 2001;12(1):47-57.

3. Auffarth GU, Wesendahl TA, Brown SJ, Apple DJ. Are there acceptable anterior chamber intraocular lenses for clinical use in the 1990s? An analysis of 4104 explanted anterior chamber intraocular lenses. Ophthalmology. 1994;101(12):1913-22. 
4. McCannel MA. A retrievable suture idea for anterior uveal problems. Ophthalmic Surg. 1976; 7(2):98-103

5. Sieprs SB. The closed chamber slipping suture technique for iris repair. Ann Ophthalmol. 1994; 26(3):71-72

6. Condon CP. Simplified small-incision peripheral iris fixation of an Acrysof intraocular lens in the absence of capsular support. J Cataract Refract Surg. 2003;29(9):1663-7.

7. Malbran ES, Malbran E Jr, Negri I. Lens guide suture for transport and fixation in secondary IOL implantation after intracapsular extraction. Int Ophthalmol. 1986 ; 9(2-3):151-60.

8. Zeh WG, Price FW. Íris fixation of posterior chamber intraocular lenses. J Cataract Refract Surg. 2000;26(7):1028-34.

9. Condon GP, Masket S, Kranemann C. Small-incision íris fixation of foldable intraocular lenses in the absence of capsule support. Ophthalmology. 2007;114(7):1311-8.

10. Navia-Aray EA. Suturing a posterior chamber intraocular lens to the iris through limbal incision: results in 30 eyes. $\mathbf{J}$ Refract Corneal Surg. 1994;10(5):565-70.

11. Höh H, Ruprecht K, Nikoloudakis N, Palmowski A. Preliminary results following implantation of íris-suture-fixated posterior-chamber lenses. Ger J Ophthalmol. 1993;2(2):70-5.

12. Zeh WG, Price FW Jr. Iris fixation of posterior chamber intraocular lenses. J Cataract Refract Surg. 2000; 26(7):1028-34
13. Wagoner MD, Cox TA, Ariyasu RG, Jacobs DS, Karp CL; American Academy of Ophthalmology. Intraocular lens implantation in the absence of capsular support. A report by the American Academy of Ophthalmology. Ophthalmology. 2003; 110(4):840-59.

14. Kaiura TL, Seedor JA, Koplin RS, Rhee MK, Ritterband DC. Complication arising from íris-fixated posterior chamber intraocular lense. J Cataract Refract Surg. 2005;31(12):2420-2.

15. Stutzman RD, Stark WJ. Surgical technique for suture fixation of an acrylic intraocular lens in the absence of capsule support. J Cataract Refract Surg. 2003;29(9):1658-62.

16. Kopel AC, Carvounis PE, Hamill MB, Weikert MP, Holz ER. Iris-sutured intraocular lenses for ectopia lentis in children. J Cataract Refract Surg. 2008 ;34(4):596-600.

\section{Endereço para correspondência \\ Ana Luiza Lise Ferreira \\ Rua Alagoas, 336, ap 31 - Higienópolis \\ CEP: 01242-902 - São Paulo-SP \\ Tel: (11) 36630139 / 93038873}

